\title{
Effect of mushroom (Schizophyllum spp.) derived $\beta$-glucan on low-fiber diet induced gut dysbiosis
}

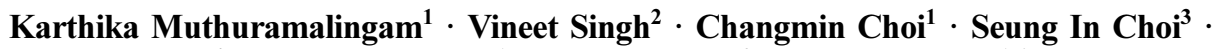 \\ Sanggyu Park ${ }^{4}$ - Young Mee Kim ${ }^{1}$ Tatsuya Unno ${ }^{2,5} \cdot$ Moonjae Cho ${ }^{1,6}$ (D)
}

Received: 22 May 2019 / Accepted: 7 June 2019 / Published Online: 30 June 2019

(C) The Korean Society for Applied Biological Chemistry 2019

\begin{abstract}
Dietary pattern has paramount importance in shaping the gut microbiota and its associated host health. Herein this study, long term (12 weeks) impact of mushroom derived dietary fiber, $\beta$-glucan, is investigated for its effect on low fiber diet consumption. Inclusion of dietary fiber into the low fiber diet (LFD) increased the abundance of genera Lactobacillus and Anaerostipes, the microbes responsible for butyrate (major 'fuel source' of colonocytes) production. Mice fed LFD with $\beta$-glucan showed significant increase in the length of small intestine compared to that of the LFD group without $\beta$-glucan. Further, dietary fiber consumption enhanced goblet cell density along with mucosal layer thickness. These results indicate promising effects of $\beta$-glucan towards maintenance of healthy gut and gut microbiota.
\end{abstract}

Moonjae Cho $(\triangle)$

E-mail: moonjcho@jejunu.ac.kr

Tatsuya Unno $(\square)$

E-mail: tatsu@jejunu.ac.kr

${ }^{1}$ Department of Biochemistry, School of Medicine, Jeju National University, Jeju 63241, Republic of Korea

${ }^{2}$ Faculty of Biotechnology, College of Applied Life Sciences, SARI, Jeju National University, Jeju 63243, Republic of Korea

${ }^{3}$ Department of Pharmaceutical Research Institute, Quegen Biotech Co. Ltd., Seoul 429931, Republic of Korea

${ }^{4}$ Division of Life \& Environmental Science, Daegu University, Daegu $712-$ 714, Republic of Korea

${ }^{5}$ Subtropical/Tropical Organism Gene Bank, Jeju National University, Jeju 63243, Republic of Korea

${ }^{6}$ Department of Biochemistry, School of Medicine, Institute of Medical Sciences, Jeju National University, Jeju 63241, Republic of Korea

This is an Open Access article distributed under the terms of the Creative Commons Attribution Non-Commercial License (http://creativecommons. org/licenses/by-nc/3.0/) which permits unrestricted non-commercial use, distribution, and reproduction in any medium, provided the original work is properly cited.
Keywords $\beta$-glucan · Dietary fiber · Gut Dysbiosis · Gut microbiota - Low-fiber diet

\section{Introduction}

Diet is one of the major factors that modulate gut microbiome. With the rapid changing lifestyle toward westernization, such as high consumption of calories, refined carbohydrates, sugar, salt, fat, animal proteins, energy dense and highly processed foods etc., the gut microbiota can dramatically get altered, leading to gut dysbiosis. Further, diet components lacking microbiota-accessible carbohydrates ensue irremediable loss of gut microbial population, thereby contributing dysfunctions, chronic inflammatory disorders, metabolic syndromes etc [1]. The susceptibility to these diseases, at-least in part, can be prevented by adding dietary fibers to the diet, thereby establishing a healthy host-microbe relationship and host immunity.

Dietary fiber, as defined by the Institute of Medicine (US), consists of non-digestible carbohydrate and lignin that are intrinsic and intact in plants [2]. On an average, the recommended intake of fiber content for a young man and woman is around $38 \mathrm{~g} /$ day and $25 \mathrm{~g} /$ day respectively [3]. Diets that lack the required amount of fibers will make the people more prone towards obesity, heart disorders, diabetes, bowel cancers, constipation etc. Foods such as whole-grain cereals, fruits, vegetables, legumes etc. are said to be rich in fiber content [4]. Consumption of dietary fibers such as cellulose, arabinoxylan, inulin, soluble corn fiber, resistant starch, guar gum, gum arabic etc., is found to be inversely linked with the risk of microbiome-associated non-communicable disorders such as obesity, cardiovascular diseases, allergies, cancer, inflammatory diseases etc. $[5,6]$.

$\beta$-Glucan, an indigestible- and hydro soluble- dietary fiber has shown to exhibit much physiological benefits on cholesterol reduction, weight management, cardiovascular risks, diabetes 
mellitus, metabolic syndrome etc. [7,8]. Being a major soluble fiber found in the cell walls of oats and barley endosperm, the $\beta$ glucan is commercially extracted from Saccharomyces cerevisiae and also from other sources such as Phellinus linteus or Sparassis crispa $[9,10]$. While oat and barley based $\beta$-glucan presents metabolic effects such as postprandial glycemic response, fungi derived $\beta$-glucan exerts immunological modulation; thereby making the sources of $\beta$-glucan as one of the prime factors contributing to its clinical outcome [11,12]. Further, the fermentation of this indigestible fiber in the lower gastrointestinal tract is said to alter the gut microbial composition, whose metabolites is found to have prime importance on host health [13,14].

Herein this study, we aimed to investigate the long-term impact of mushroom derived $\beta$-glucan, a soluble dietary fiber consumption on the gut dysbiosis induced by low fiber consumption in animal model.

\section{Materials and Methods}

\section{Animal care}

C57BL/6J male mice (5 weeks old) were used in this study. With a laboratory acclimation period of 1 week, the mice were 6 weeks old when they were subjected for experimentation. $12 \mathrm{~h} / 12 \mathrm{~h}$ of light/dark cycle with ambient atmosphere was provided to the mice. All the animals were taken care of according to the rules framed by Animal Care and Use Committee (ACUC No: 20180018).

\section{Experimental design}

The experimental scheme was given in Scheme 1. Four experimental groups with $n=11$ mice per group was classified as: normal diet group (ND), low fiber diet group-AIN 76A (LFD), LFD with $3 \mathrm{~g}$ / $\mathrm{kg}$ of $\beta$-glucan (LFD $+\mathrm{BG1}$ ) and LFD with $5 \mathrm{~g} / \mathrm{kg}$ of $\beta$-glucan (LFD + BG2). $\beta$-Glucan ( $\beta-1,6 / 1,3$ glucan isolated from Schizophyllum spp. with average molecular weight of $1.78 \sim 1.79 \times 10^{6} \mathrm{Da}$ ) was provided by Quegen Biotech Co. Ltd., Republic of Korea. During the experimental study period, mice were provided with $a b$ libitum of water and corresponding diets. At two time points in the scale of 12 weeks experimental period ( $6^{\text {th }}$ week and $12^{\text {th }}$ week), feces from individual mice in all the groups were collected and subjected for microbiota analysis. On the day prior to the sacrifice of mice, they were left starving through the night. The mice were orally administered $100 \mu \mathrm{L}$ of activated charcoal solution prepared in $1 \mathrm{X}$ PBS, 20 min prior to sacrificing it. The distance travelled by the charcoal in the intestine was observed and the intestinal transit rate was calculated accordingly: Intestinal transit rate $=($ Distance travelled by the activated charcoal/Length of the small intestine) $\times 100$. Once the mice were sacrificed, the internal organs were isolated and weighed. Small intestine samples collected from the mice were fixed using $4 \%$ formaldehyde solution for further histopathological analysis.

\section{Physiological measurements}

At the start of every week, measurement on body weight, weekly consumption of food and water were measured. In addition, 25 pieces of feces from each cage was collected and weighed. The fecal moisture content was calculated by weighing the difference in feces weight before and after drying at $60^{\circ} \mathrm{C}$ for 2 days.

\section{Histopathological analysis}

The formaldehyde fixed small intestine samples were sectioned and subjected to Alcian blue and PAS staining, in-order to observe the effect of different diets on intestinal architecture and mucin abundance. The stained sectioned were imaged using Olympus BX51 microscope. The thickness of mucus layer is quantified using ImageJ.

\section{Fecal microbiota analysis}

Feces from individual mice were collected on $6^{\text {th }}$ week and $12^{\text {th }}$ week of the diet-based experimental study. 16S rRNA targeted PCR amplification was done to the QIAamp, PowerFecal DNA kit extracted total DNA samples and the sequenced data (sequencing was performed by Macrogen, Seoul, Republic of Korea) was processed using MOTHUR [15], a bioinformatics software package. Briefly, raw sequence reads assembled using make.contigs command were aligned to SILVA database using align.seqs, followed by trimming the rare sequences using split.abund and pre.cluster. Further, chimeric sequences were removed using chimera.vsearch command and non-bacterial sequences such as mitochondria and chloroplasts were removed before clustering the resulted clean reads using opti.clust at the distance 0.03 . Nonmetric multidimensional scaling (NMDS), using Bray-curtis dissimilarity distance, computed with nmds MOTHUR subroutine was employed to identify the bacterial Operational Taxonomic Units (OTUs).

\section{Statistical analysis}

Values are given as mean \pm standard error and the statistical significance was calculated using Student t-test. Significance in the microbial population difference was examined using Analysis of molecular variance (AMOVA).

\section{Results and Discussion}

Compared to that of the normal diet, mice fed with low fiber diet showed a relative $10 \%$ increase in body weight (Fig. 1A, B). This result gets reflected with the increase in the Firmicutes/Bacteroidetes ratio, a "obese type microbiota from the fecal analysis (shown in Fig. S3A). In general, high fiber intake is associated with weight management by increasing gastric distension and satiety, whereas poor-fiber diet enhances hunger thereby increasing total energy intake resulting in overweight and obesity. Although not significant ( $p>0.05$ ), inclusion of $\beta$-glucan to LFD exhibits promising result 

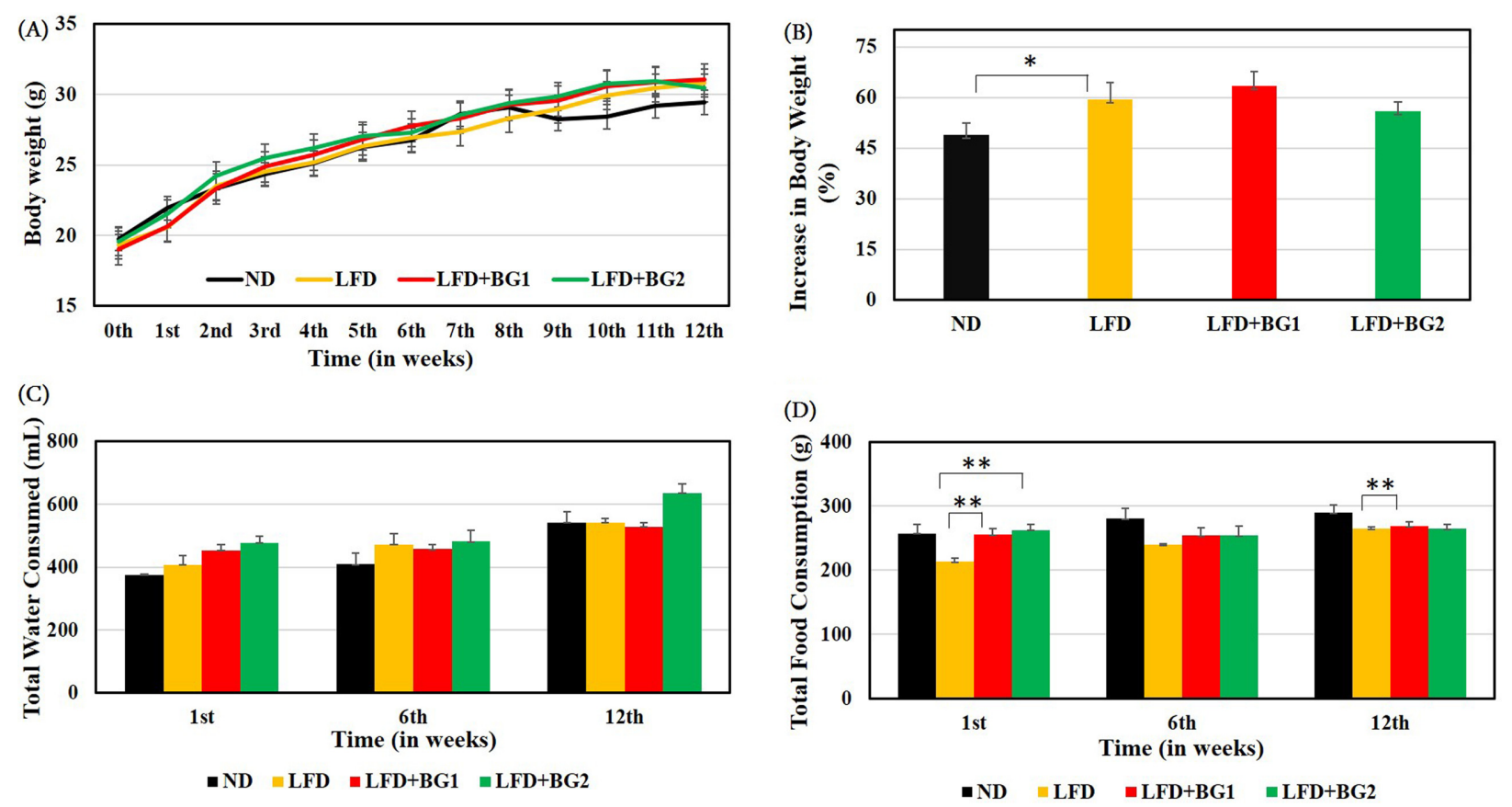

Fig. 1. Effect of low fiber diet with/without $\beta$-glucan on physiological parameters of the mice. (A) Body weight; (B) percentage increase in body weight; (C) water consumption per week; and (D) food consumption per week
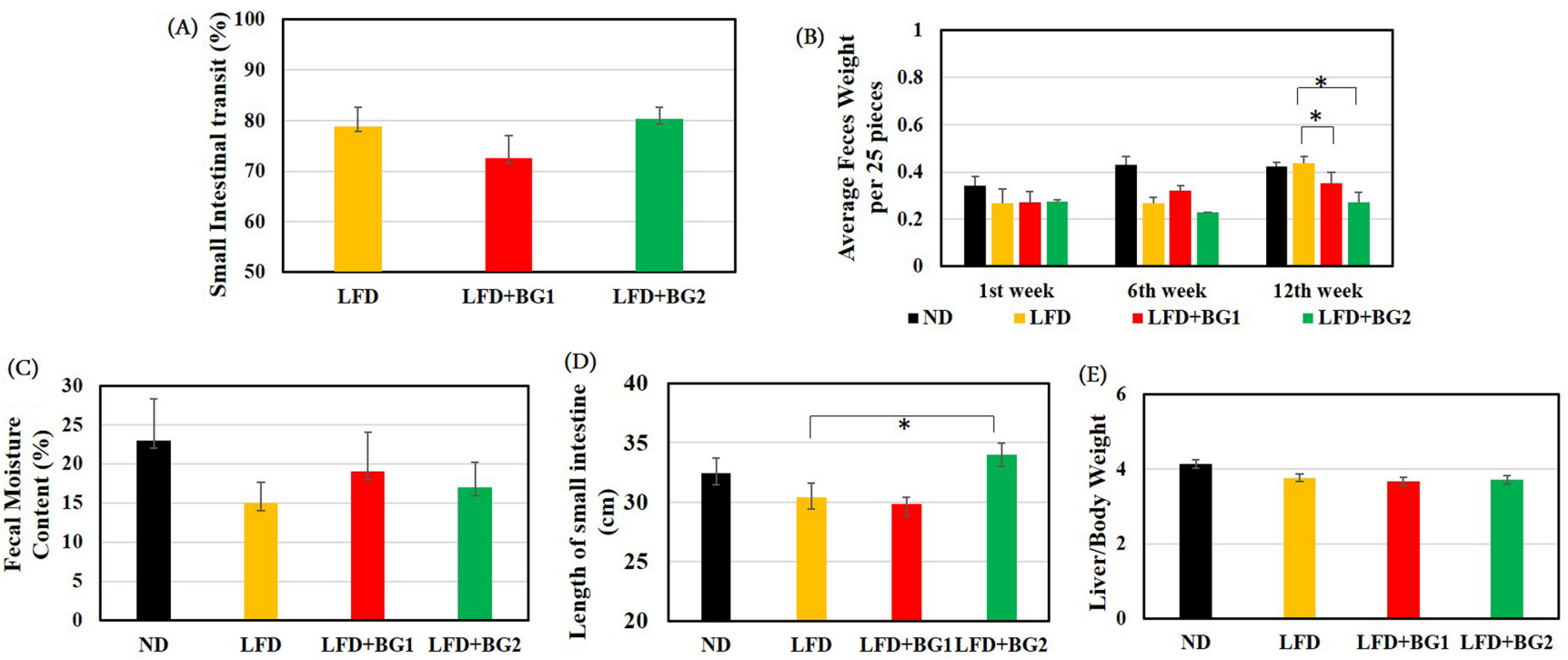

Fig. 2. Effect of low fiber diet with/without $\beta$-glucan on (A) intestinal transit rate; (B) feces weight; (C) fecal moisture content; (D) length of small intestine; and (E) Liver/body weight

towards weight reduction. Mice fed with LFD + BG2 showed relatively higher water consumption than the rest of the groups (Fig. 1C) while there was no significant difference in the food consumption (Fig. 1D) among the low fiber diet groups (LFD, $\mathrm{LFD}+\mathrm{BG} 1, \mathrm{LFD}+\mathrm{BG} 2)$ at the $6^{\text {th }}$ and $12^{\text {th }}$ week of the experimental period.

LFD and LFD + BG2 showed almost similar transit percentage (Fig. 2A), however, it was reduced with LFD + BG1. Fecal weight is one of the key indicators of intestinal health [16]. There was a significant fecal weight reduction in the $\beta$-glucan included LFD groups compared to that of the LFD control group in a dose dependent manner (Fig. 2B). Thus, mushroom derived $\beta$-glucan doesn't have much impact on bowel movement associated intestinal health. In addition, the mice fed with LFD showed lowest moisture content in the fecal samples (Fig. 2C); whereas the dietary fiber included LFD groups showed relatively higher 


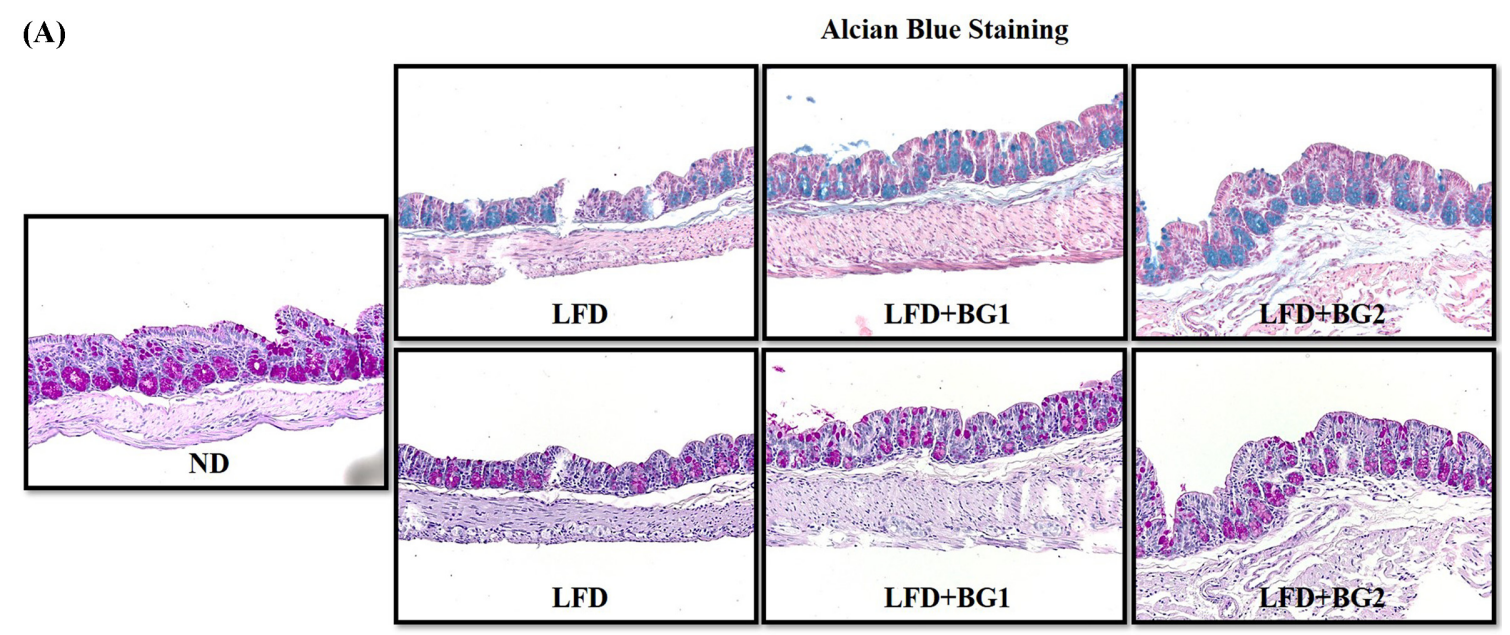

PAS Staining

(B)

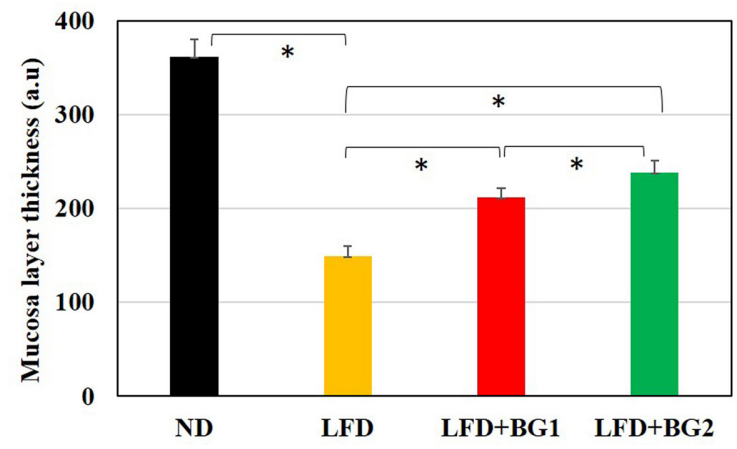

Fig. 3. Effect of low fiber diet with/without $\beta$-glucan on histological analysis of intestinal sample (A) Alcian blue and PAS staining; and (B) mucosa layer thickness

moisture content. This leads to conclude that the reduction in fecal weight doesn't come from its moisture content and further investigation on other factors such bacterial biomass, protein or nitrogenous or undigested dietary matters is required. Mice fed with higher concentration of $\beta$-glucan incorporated LFD showed increase in small intestine length (Fig. 2D). This may aid in higher nutrient absorption by the increased cross-sectional area of intestinal mucosal barrier. However, no significant changes were observed in the liver/body weight of the low fiber diet groups (Fig. 2E).

Fiber-deprived diets urge the gut microbiota to rely on the 'highly glycosylated' intestinal mucus layer, whose primary function is to protect the underlying gastrointestinal tract (comprising of epithelial cells) from bacterial invasion [17,18]. From the histopathological staining of the small intestine sample (Fig. 3A), it was observed that $\beta$-glucan incorporated LFD groups showed enhanced goblet cell count with significantly higher mucosal layer thickness (dose-dependent increase) (Fig. 3B) and mucosasupporting submucosal layer than the LFD control group, thereby presaging that consumption of diet lacking in fiber content may result in bacterial invasion in the mucus layer, leading to 'the leaky' gut wall; whereas inclusion of dietary fiber will ameliorate the condition. Though not significant, we observed a dose dependent decrease in serum triglyceride level together with enhanced highdensity lipoprotein level in the $\beta$-glucan incorporated LFD groups than the LFD group (Fig. S1).

For microbiota analysis, 19,590 reads were randomly sampled from each fecal sample to normalize the number of reads. More than $99 \%$ Good's coverage was observed in each sample; thus, this random sampling hardly affected the number of observed species. Results in Figure S2 showed that the use of low-fiber diet (LFD) significantly decreased species richness and evenness. In this study, 12th week samples showed lower richness and evenness compared to those at the 6th week. Since dietary fibers are the source of energy for bacteria in the large intestine, lack of this fiber in diet could cause reduction of certain species that feed on dietary fibers. Previously, it has been reported that dietary fibers are fermented by the intestinal bacteria to produce short chain fatty acids such as butyrate and propionate, resulting in improving the intestinal immune system and host's health [1].

Results in Fig. 4 show that the dysbiosis caused by LFD are more obvious at the 12th week than at the 6th week as NMDS plots were more scattered. On the other hand, LFD + BG1 samples are less scattered and plotted relatively closer to the 


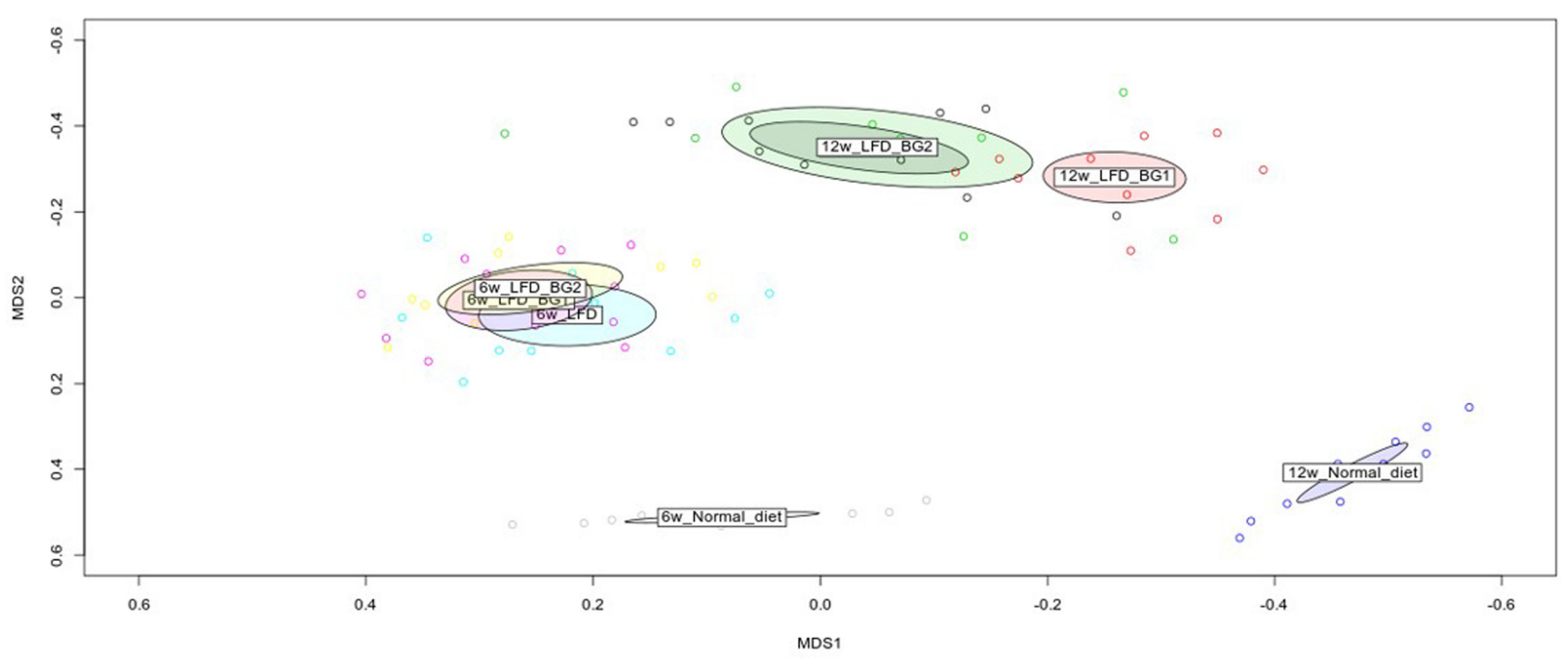

Fig. 4. Analysis of microbial community comparison by non-metric multidimensional scaling (NMDS)

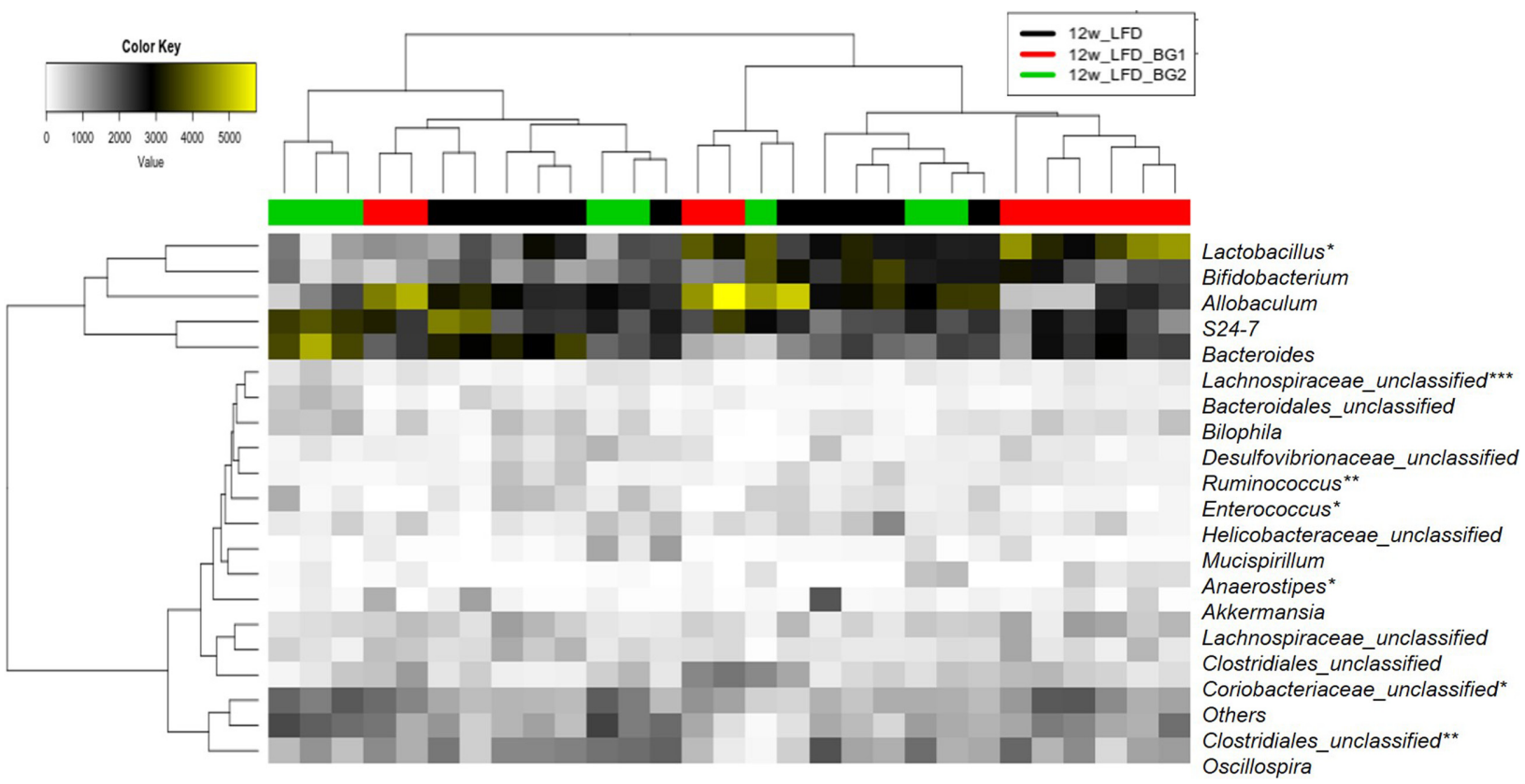

Fig. 5. Comparison of the taxonomic composition at the genus level. *, **, and *** indicate significant difference between LFD and LFD + BG1, LFD and LFD+BG2, and LFD and both LFD+ BG1 and LFD + BG2, respectively

normal diet at the 12th week, suggesting that LFD + BG1 may have slightly ameliorated gut dysbiosis. While distribution of LFD + BG2 samples were similar to that of LFD samples at the 12th week, LFD + BG1 showed significantly different microbiota distribution when compared with that of LFD samples $(p<0.001)$. These shifts observed in LFD + BG1 group were not observed at the phylum level (Fig. S3A). At the family level, the lower abundance of the family S24-7 was observed in LFD, suggesting that lack of fiber cause a decrease in the abundance of S24-7 (Fig. S3B). While distribution at the family level for normal diet showed a clear separation between the 6th and 12th week, LFD groups did not, suggesting that unbalanced intestinal microbiota among LFD groups. At the 12th week, LFD + BG1 samples were clustered due to the increased abundance of genera, Lactobacillus, and Anaerostipes (Fig. 5). The genus Lactobacillus is a wellknown probiotic bacterium, while some species of the genus Anaerostipes are known to produce butyrate by utilizing lactate[19,20], suggesting that the increase of these two genera may cross-feed to produce butyrate in the gut. The roles of butyrate produced in the gut include being the major energy source for colonocytes, maintaining the colonial mucosal health, and regulating the intestinal tight junctions along with imparting 
(A)

$$
\text { 12w_LFD vs 12w_LFD_BG1 }
$$

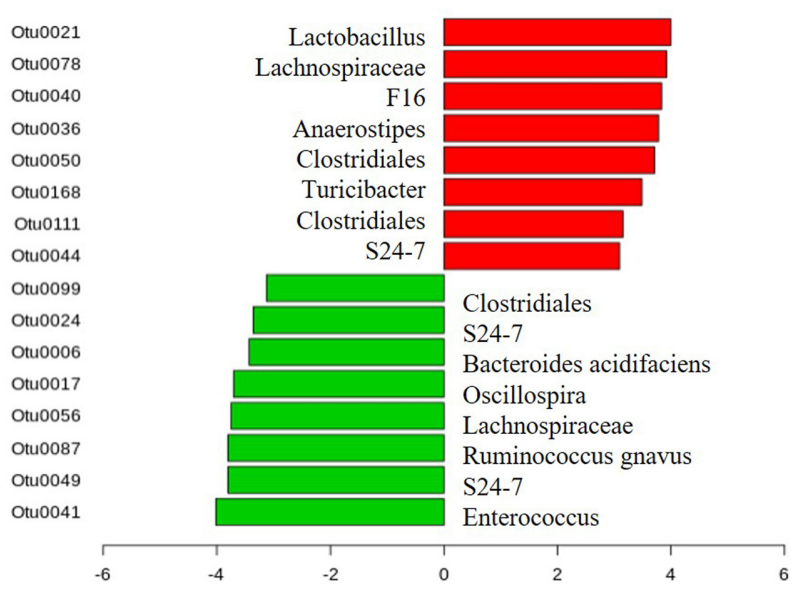

12w_LFD vs 12w_LFD_BG2

Otu0008
Otu0050
Otu0162
Otu0040
Otu0036
Otu0179
Otu0079
Otu0131
Otu0152
Otu0127
Otu0072
Otu0151
Otu0083
Otu0019
Otu0174
Otu0044
Otu0117
Otu0249
Otu0130
Otu0024
Otu0058
Otu0087
Otu0056
Otu0017
Otu0021

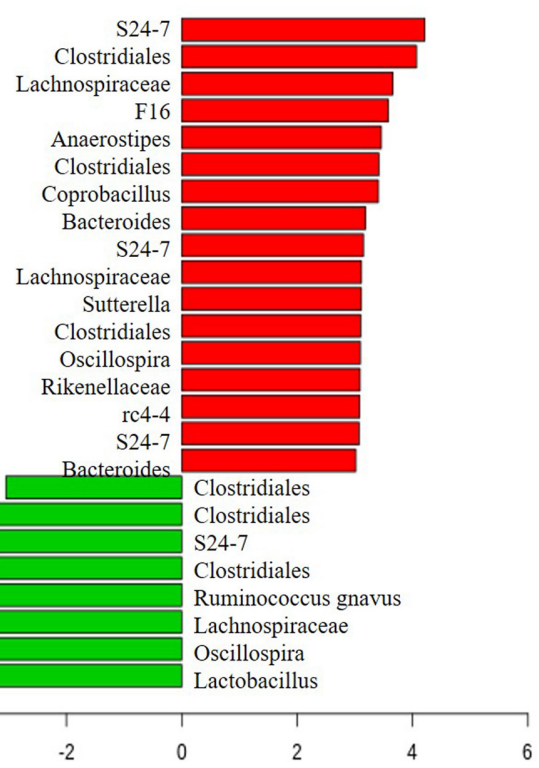

Fig. 6. Differential abundance analysis between (A) LFD and LFD+BG1; and (B) LFD and LFD + BG2 among the 12th-week samples $(p<0.05$, LDA >3) (OTU-Operational Taxonomic Unit]

immunomodulatory and anti-inflammatory properties on the gut barrier [21,22]. Therefore, our results suggest that long-term use of $\beta$-glucan may shift microbiota toward preferred environment for butyrate production in the gut. It should be noted that the higher dose of $\beta$-glucan did not increase Lactobacillus, thus beneficial effects of $\beta$-glucan may be dose-independent.

Figure 6 show differentially abundant OTUs between LFD samples and LFD + BG samples. There are 3 OTUs (0040, 0036, and 0050) increased and 4 OTUs (0024, 0017, 0056, and 0087) decreased in both BG groups, indicating that $\beta$-glucan doseindependently increased Anaerostipes, unknown genera in F16 and Clostridiales and decreased Oscillospira, Ruminococcus gnavus, and unknown genera in S24-7 and Lachnospiracea. The abundance of Oscillospira, Ruminococcus, and Lachnospiracea is highly correlated with the pathogenesis of Type 1 Diabetes by decreasing FoxP $3^{+}$regulatory $\mathrm{T}$ cells [23]. Herein our study, the LFD + BG groups has the tendency to decrease their abundance thereby exhibiting fiber restricted diet associated anti-diabetic effect. It is interesting that the abundance of Lactobacillus was increased in LFD + BG1 but decreased in LFD + BG2. As there are more OTUs that were significantly increased in LFD + BG2 group, some of these OTUs may have competed against Lactobacillus over substrates, or the decrease of some OTUs in LFD + BG1 may have helped Lactobacillus to increase. Further study is needed to investigate who competes against whom over substrates in the gut. This is important to maintain the healthy gut microbiota and it should be noted that the dose determined based on mice experiment should not be applied to human case as previously reported [24].
To summarize, the use of $\beta$-glucan improves LFD induced gut dysbiosis in terms of goblet cell production and enhanced mucosa layer protective barrier. LFD consumption significantly changed gut microbiota compared to that of a normal diet. While the changes were seen in increased Firmicutes/Bacteroidetes ratio, it was rather random at the family level, suggesting that the use of LFD disrupt the healthy gut microbiota in various ways. The lower dose of $\beta$-glucan increased Lactobacillus and Anaerostipes, which may have contributed to stabilizing the gut microbiota, while a higher dose of $\beta$-glucan decreased Lactobacillus thus failed to stabilize the gut microbiota. Further studies are needed to find the optimum dose of $\beta$-glucan toward human application, but here, we report the promising effects of $\beta$-glucan in maintaining healthy gut microbiota.

Acknowledgment This research was supported by the 2019 scientific promotion program funded by Jeju National University

\section{Conflict of interest}

The authors declare no conflict of interest

\section{References}

1. Makki K, Deehan EC, Walter J, Backhed F (2018) The Impact of Dietary Fiber on Gut Microbiota in Host Health and Disease. Cell Host and Microbe 23: 705-715. doi: 10.1016/j.chom.2018.05.012

2. National Research Council (U.S.), Institute of Medicine (U.S.). Standing Committee on the Scientific Evaluation of Dietary Reference Intakes. Institute of Medicine (U.S.). Panel on the Definition of Dietary Fiber. 
(2001) Dietary reference intakes: proposed definition of dietary fiber: a report of the Panel on the Definition of Dietary Fiber and the Standing Committee on the Scientific Evaluation of Dietary Reference Intakes

3. Slavin JL (2005) Dietary fiber and body weight. Nutrition 21: 411-418. doi: 10.1016/j.nut.2004.08.018

4. Marlett JA, McBurney MI, Slavin JL (2002) Health implications of dietary fiber. Journal of the American Dietetic Association 102: 993 1000

5. King DE, Mainous AG, Lambourne CA (2012) Trends in Dietary Fiber Intake in the United States, 1999-2008. Journal of the Academy of Nutrition and Dietetics 112: 642-648. doi: 10.1016/j.jand.2012.01.019

6. Deehan EC, Walter J (2016) The Fiber Gap and the Disappearing Gut Microbiome: Implications for Human Nutrition. Trends in Endocrinology and Metabolism 27: 239-242. doi: 10.1016/j.tem.2016.03.001

7. El Khoury D, Cuda C, Luhovyy BL, Anderson GH (2012) Beta glucan: Health benefits in obesity and metabolic syndrome. Journal of Nutrition and Metabolism 2012:. doi: 10.1155/2012/851362

8. Xin-Zhong H, Xia-Lu S, Xiao-Ping L, Liu L, Jian-Mei Z, Zing-Yun C (2015) Effect of dietary oat $\beta$-glucan on high-fat diet induced obesity in HFA mice. Bioactive Carbohydrates and Dietary Fibre 5: 79-85. doi: 10.1016/j.bcdf.2014.12.006

9. Kim SY, Song HJ, Lee YY, Cho KH, Roh YK (2009) Biomedical Issues of Dietary fiber $\beta$-Glucan. Journal of Korean Medical Science 21: 781. doi: $10.3346 / \mathrm{jkms} .2006 .21 .5 .781$

10. Choi JS, Kim H, Jung MH, Hong S, Song J (2010) Consumption of barley $\beta$-glucan ameliorates fatty liver and insulin resistance in mice fed a high-fat diet. Molecular Nutrition and Food Research 54: 1004-1013. doi: 10.1002/mnfr.200900127

11. de Araújo TV, Andrade EF, Lobato RV, Orlando DR, Gomes NF, de Sousa RV, Zangeronimo MG, Pereira LJ (2017) Effects of beta-glucans ingestion (Saccharomyces cerevisiae) on metabolism of rats receiving high-fat diet. Journal of Animal Physiology and Animal Nutrition 101: 349-358

12. Miyamoto J, Watanabe K, Taira S, Kasubuchi M, Li X, Irie J, Itho H, Kimura I (2018) Barley $\beta$-glucan improves metabolic condition via shortchain fatty acids produced by gut microbial fermentation in high fat diet fed mice. PLoS ONE 13: 1-13. doi: 10.1371/journal.pone.0196579

13. Wang Y, Ames NP, Tun HM, Tosh SM, Jones PJ, Khafipour E (2016) High molecular weight barley $\beta$-glucan alters gut microbiota toward reduced cardiovascular disease risk. Frontiers in Microbiology 7: 1-15. doi: 10.3389/fmicb.2016.00129

14. Ghaffarzadegan T, Zhong Y, Fåk Hållenius F, Nyman M (2018) Effects of barley variety, dietary fiber and $\beta$-glucan content on bile acid composition in cecum of rats fed low- and high-fat diets. Journal of Nutritional Biochemistry 53: 104-110. doi: 10.1016/j.jnutbio.2017.10.008
15. Schloss PD, Westcott SL, Ryabin,T, Hall JR, Hartmann M, Hollister EB, Lesniewski RA, Oakley BB, Parks DH, Robinson CJ, Sahl JW, Stres B, Thallinger GG, Van Horn JD, Weber CF (2009) Introducing mothur: Open-Source, Platform-Independent, Community-Supported Software for Describing and Comparing Microbial Communities. Applied and Environmental Microbiology 75: 7537-7541. doi: 10.1128/AEM.0154109

16. de Vries J, Miller PE, Verbeke K (2015) Effects of cereal fiber on bowel function: A systematic review of intervention trials. World journal of gastroenterology 21: 8952-8963. doi: 10.3748/wjg.v21.i29.8952

17. Hansson GC (2012) Role of mucus layers in gut infection and inflammation. Current Opinion in Microbiology 15: 57-62. doi: 10.1016/ j.mib.2011.11.002

18. Desai MS, Seekatz AM, Koropatkin NM, Kamada N, Hickey CA, Wolter M, Pudlo NA, Kitamoto S, Terrapon N, Muller A, Young VB, Henrissat B, Wilmes P, Stappenbeck TS, Nunez G, Martens EC, (2016) A Dietary Fiber-Deprived Gut Microbiota Degrades the Colonic Mucus Barrier and Enhances Pathogen Susceptibility. Cell 167: 1339-1353.e21. doi: 10.1016/j.cell.2016.10.043

19. Sato T, Matsumoto K, Okumura T, Yokoi W, Naito E, Yoshida Y, Nomoto K, Ito M, Sawada H (2008) Isolation of lactate-utilizing butyrate-producing bacteria from human feces and in vivo administration of Anaerostipes caccae strain L2 and galacto-oligosaccharides in a rat model. FEMS Microbiology Ecology 66: 528-536. doi: 10.1111/j.15746941.2008.00528.x

20. Bui TPN, de Vos WM, Plugge CM (2014) Anaerostipes rhamnosivorans sp. nov., a human intestinal, butyrate-forming bacterium. International Journal of Systematic and Evolutionary Microbiology 64: 787-793. doi: 10.1099/ijs.0.055061-0

21. Hamer HM, Jonkers D, Venema K, Vanhoutcin S, Troost FJ, Brummer RJ (2008) Review article: The role of butyrate on colonic function. Alimentary Pharmacology and Therapeutics 27: 104-119. doi: 10.1111/ j.1365-2036.2007.03562.x

22. Monolayers C-C, Peng L, Li Z, Green RS, Holzman IR, Lin J (2009) Butyrate Enhances the Intestinal Barrier by Facilitating Tight Junction Assembly via Activation of AMP-Activated Protein Kinase. J Nutr 139: 1619-1625. doi: 10.3945/jn.109.104638.1619

23. Krych, Nielsen DS, Hansen AK, Hansen CHF (2015) Gut microbial markers are associated with diabetes onset, regulatory imbalance, and IFN-ã level in NOD Mice. Gut Microbes 6: 101-109. doi: 10.1080/ 19490976.2015.1011876

24. Nguyen TLA, Vieira-Silva S, Liston A, Raes J (2015) How informative is the mouse for human gut microbiota research? Disease Models \& Mechanisms 8: 1-16. doi: 10.1242/dmm.017400 